\title{
Neurological Disease Associated With Enterovirus Infection: Retrospective Analysis of Risk Factors in a Pediatric Population
}

Eulàlia Turón Viñas ( $\nabla$ eturon@santpau.cat)

Hospital de la Santa Creu i Sant Pau https://orcid.org/0000-0001-6171-0282

Fatima Castillo Gómez

Hospital de la Santa Creu i Sant Pau

Elisenda Moliner Calderón

Hospital de la Santa Creu i Sant Pau

Laura Armendáriz Lacasa

Hospital de la Santa Creu i Sant Pau

Emma Carbonell Estarellas

Hospital de la Santa Creu i Sant Pau

Margarita del Cuerpo Casas

Hospital de la Santa Creu i Sant Pau

Nuria Rabella Garcia

Hospital de la Santa Creu i Sant Pau

\section{Research Article}

Keywords: Enterovirus, neurological disease, encephalitis, aseptic meningitis, EV-A71, children

Posted Date: January 24th, 2022

DOI: https://doi.org/10.21203/rs.3.rs-1258816/v1

License: (9) (i) This work is licensed under a Creative Commons Attribution 4.0 International License.

Read Full License 


\title{
TITLE: NEUROLOGICAL DISEASE ASSOCIATED WITH ENTEROVIRUS INFECTION: RETROSPECTIVE ANALYSIS OF RISK FACTORS IN A PEDIATRIC POPULATION
}

Fátima Castillo Gómez. Department of Pediatrics, Hospital de la Santa Creu i Sant

Pau, Barcelona (Spain). fcastillog@santpau.cat

Eulàlia Turón Viñas. Department of Pediatrics, Child Neurology Unit, Hospital de la Santa Creu i Sant

Pau, Barcelona (Spain). eturon@santpau.cat

Elisenda Moliner Calderón. Department of Pediatrics, Infectious Diseases Unit, Hospital de la Santa

Creu i Sant Pau, Barcelona (Spain). emoliner@santpau.cat

Laura Armendariz Lacasa. Department of Pediatrics, Hospital de la Santa Creu i Sant Pau, Barcelona

(Spain). larmendariz@santpau.cat

Emma Carbonell Estarellas. Department of Pediatrics, Hospital de la Santa Creu i Sant Pau, Barcelona (Spain). ecarbonelle@santpau.cat

Margarita del Cuerpo Casas. Department of Microbiology, Virology Unit, Hospital de la Santa Creu i

Sant Pau, Barcelona (Spain). mdelcuerpo@santpau.cat

Nuria Rabella Garcia. Department of Microbiology, Virology Unit, Hospital de la Santa Creu i Sant

Pau, Barcelona (Spain). nrabella@santpau.cat

Corresponding author:Eulàlia Turón-Viñas. Department of Pediatrics, Child Neurology Unit, Hospital de la Santa Creu i Sant Pau, Barcelona (Spain). eturon@santpau.cat. ORCID ID: 0000-0001-6171-0282

\begin{abstract}
Enteroviruses are a type of RNA-strained virus with more than 100 different genotypes. Infection can be asymptomatic, or symptoms can range from mild to severe. Some patients can develop neurological involvement, such as aseptic meningitis, encephalitis, or even cardiorespiratory failure. However, in children, the risk factors for developing severe neurological involvement are not well understood. The aim of this retrospective study was to determine the risk factors associated with severe neurological involvement in children hospitalized for neurological disease after enterovirus infection. One-hundred and seven children hospitalized from 2009 to 2019 were included. Patients were classified according to the World Health Organization case definition for neurological complications in hand, foot and mouth disease. Our findings showed that, in children between age 2 and 5, the appearance of neurological symptoms within the first 12 hours from infection onset - especially if associated with skin rash-was a significant risk factor for severe neurological involvement. Detection of enterovirus in cerebrospinal fluid was more likely in patients with aseptic meningitis. By contrast, other biological samples (e.g., feces or nasopharyngeal fluids) were necessary to detect enterovirus in patients with encephalitis. The genotype most commonly associated with the most severe neurological conditions was EV-A71. E-30 was mostly associated with aseptic meningitis. Awareness of the risk factors associated with worse neurological outcomes could help clinicians to better manage these patients to avoid unnecessary admissions and/or ancillary tests.
\end{abstract}

\section{KEYWORDS}

Enterovirus, neurological disease, encephalitis, aseptic meningitis, EV-A71, children. 


\section{ABBREVIATIONS}

ANS: Autonomic Nervous System

CNS: Central Nervous System

CSF: Cerebrospinal fluid

EV: Enterovirus

EVs: Enteroviruses

HFMD: Hand, foot and mouth disease

IQR: Interquartile range

MRI: Magnetic resonance imaging

PICU: Pediatric intensive care unit

RT-PCR: Reverse transcription polymerase chain reaction

WHO: World Health Organization

\section{INTRODUCTION}

Enteroviruses (EVs) are single-strained RNA viruses that are endemic in most countries. Four different species of EV (A, B, C and D) are known to affect humans, with more than 100 different genotypes. These viruses are responsible for common viral infections in children, with most $(90 \%)$ being mild or asymptomatic. However, in some cases, infection can lead to more serious conditions with neurological, cardiac, or hepatic involvement [1]. Neurological involvement ranges from mild (e.g., meningitis) to severe (e.g., encephalitis or cardiopulmonary failure), with the latter conditions associated with high morbidity and mortality rates. Given the potential risks, early diagnosis of EV infections in children at high risk of developing the most severe forms is crucial [1]. While most EV genotypes can cause neurological symptoms, some appear to have higher affinity for the central nervous system (CNS). Meningitis is the most common neurological disease associated with EV infections, accounting for up to $90 \%$ of EVassociated neurological conditions; by contrast, encephalitis is much rarer, observed in less than $3 \%$ of EV infections ].

EVs are detected in $50 \%-80 \%$ of patients with aseptic meningitis [3-5]. Of the genotypes isolated in the cerebrospinal fluid (CSF) of patients with meningitis, the most common is echovirus 30 (E30), which has been associated with meningitis and meningoencephalitis outbreaks around the world [6,7]. E30 was isolated in more than $30 \%$ of children diagnosed with meningitis from 2002 to 2012 in East China [8].

In recent years, EV A71 (EV-A71) and D68 (EV-D68) have received substantial attention due to their association with specific forms of severe neurological disease. EV-A71 has been associated with hand, foot, and mouth disease (HFMD) outbreaks in Asia, leading to neurological disease in some children. EV-A71 infection has also been associated with a high incidence of neurological symptoms in Europe [9]. EV-A71 enters the brain due to retrograde axonal transport through cranial or peripheral nerves, usually up to five days from infection onset. The initial presentation is usually nonspecific and includes headache, malaise, 
weakness, and irritability, which may later be followed by other symptoms such as lethargy, limb tremor, nausea, and/or myopathy [10]. Most patients recover without any sequelae; however, some children worsen and develop ataxia, myoclonus, or lower cranial nerve palsy, suggesting cerebellar and/or brain stem involvement. The most severe forms can lead to dysregulation of the autonomic nervous system (ANS) with arterial hypertension, cardiac arrhythmia and cardiopulmonary failure. These conditions seem to be triggered by a cytokine storm leading to pulmonary edema and myocardial dysfunction [11]. Without proper diagnosis and early treatment, EV-associated HFMD can be fatal in some cases and survivors may have severe sequelae [12]. The World Health Organization (WHO) has developed clinical guidelines for the management of HFMD, classifying neurological involvement as follows: aseptic meningitis, meningitis, brainstem encephalitis, encephalomyelitis, acute flaccid paralysis, pulmonary edema, and cardiopulmonary failure [13]. Treatment will depend on the specific neurological disease, and may include immunoglobulins, corticosteroids, and/or plasma exchange in more serious forms [14].

In an outbreak that occurred in Spain (mostly in the region of Catalonia) in 2016, approximately 100 cases of encephalomyelitis and brain stem encephalitis associated with EV-A71 infection were reported [15-17]. Another study reported some additional cases of EV-A71 infection in children in that same year (2016) in Spain, but without neurological involvement [15]. This genotype was previously detected in other European 
countries (Germany and France) [18], and later (2018) in the United States, although these outbreaks were milder than those in Spain [19].

Despite the potential severe consequences of EV infection, little is known about the risk factors for severe neurological involvement in children. In this context, the objective of the present retrospective study was to evaluate the clinical and ancillary characteristics of patients hospitalized for EV infection from 2009 to 2019 (before and after the 2016 outbreak) in a hospital in Catalonia in order to identify risk factors associated with the development of neurological involvement.

\section{MATERIALS AND METHODS}

\section{Study design}

This was a retrospective, observational study of children (< age 18) diagnosed with EV-associated neurological disease at the Hospital de la Santa Creu i Sant Pau in Barcelona (Catalonia), Spain. The study period was from January 2009 to July 2019.

Case definition was the presence of neurological symptoms and a confirmed EV from a biological sample (CSF, feces, nasopharyngeal fluids). Patients with febrile seizures without other neurological involvement were excluded.

To detect and isolate EV in nasopharyngeal and feces samples, cell cultures in different lines (MRC-5, A549, HEp2 and RD) were used in accordance with WHO recommendations for the detection of poliomyelitis. To check for the presence of EV in CSF, we performed reverse transcription polymerase chain reaction (RT-PCR) assays (Cepheid®Xpert EV). All culture-isolated and all CSF-detected EVs were characterized. The patients' demographic, clinical, blood tests, microbiological and radiological data are shown in Table 1.

\section{Statistical analysis}

Descriptive data are provided. Categorical variables are expressed as number of cases and percentages and quantitative variables as means with interquartile range (p25-p75). For categorical variables, Chi-square contingency tables were prepared. The likelihood ratio as the inferential test because it can be used regardless of the number of cells. For quantitative variables we used the Student's t-test, without assuming equal variances. No multiple comparison correction was performed as this was an exploratory study to identify risk factors, which could then be used to facilitate future studies. The level of significance was set at $5 \%(\alpha=0.05)$. All analyses were performed with the IBM-SPSS (v.26) statistical software package.

For the analysis, patients were classified by age group as follows: $<3$ months old; $3-6 \mathrm{~m} ; \geq 6 \mathrm{~m}$ to $\leq 2 \mathrm{y} ; \geq$ 2 to $\leq 5 \mathrm{y} ;$ and $\geq 5$.

\section{RESULTS}

A total of 174 patients met the case definition during the study period. The mean patient age was 3.1 years (interquartile range: $0.2-5.1$ years). Patients aged 2-5 years made up the largest age group $(n=57)$, followed 
by patients $\geq$ age $5(\mathrm{n}=48)$. Most $(>80 \%)$ of the children were healthy prior to infection. The patients' characteristics are shown in Table 1.

Most of the children (85\%) presented with fever and 7.5\% had a skin rash: 4 (2.3\%) showed HFMD and 8 (4.6\%) herpangina. Twenty-five patients (14.4\%) had respiratory symptoms. In $44.8 \%$ of the children $(n=78)$ the first neurological symptoms developed within the first 12 hours of illness onset; in $10.9 \%$ of children $(\mathrm{n}=19)$, the symptoms appeared three days after onset. The most common neurological symptoms at onset were headache $(\mathrm{n}=134 ; 77 \%)$, decreased consciousness $(\mathrm{n}=18 ; 10.3 \%)$ and combined myoclonus and ataxia ( $\mathrm{n}=11 ; 6.3 \%$ ) (Table 2). Patients were classified according to their clinical signs following the WHO case definition for neurological complications of HFMD (Table 3). Aseptic meningitis was the most common disease in patients $<3$ months of age while encephalitis and brainstem encephalitis were the two most common conditions among patients in the 2-to-5-year age range.

Fifty-eight patients (33.3\%), most of whom were diagnosed with aseptic meningitis, did not require hospitalization. The other 136 patients (77.7\%) required hospitalization (mean duration: 4.5 days; IQR: 15 days). The most common reasons for admission were decreased consciousness, signs of neurological involvement, and age $<3$ months. The mean duration of admission was longer in infants $<3$ months of age (9.6 days; IQR: 3.7-15.4) with respect to other age groups. Twenty-three patients (13.2\%) required admission to the pediatric intensive care unit (PICU) due to status epilepticus ( $n=4)$ or severe encephalopathy $(\mathrm{n}=19)$. Six children required mechanical ventilation and four of these patients received inotropic drugs.

Twenty-eight patients (16.1\%) underwent magnetic resonance imaging (MRI), with abnormal findings in 9 cases: rhombencephalitis (9/9) and myelitis (6/9). In the other children, the MRI results were normal.

A total of 306 samples were obtained from CSF ( $n=172)$, nasopharyngeal fluids ( $n=72)$, and feces $(n=62)$. EV was detected (RT-PCR) in the CSF of 142 children (81.6\%), most of whom were diagnosed with aseptic meningitis ( $\mathrm{n}=134)$. In patients with encephalitis $(\mathrm{n}=15)$, EVs were detected in five cases $(33 \%)$ and in those with brainstem encephalitis $(n=10)$, EVs were found in two patients (20\%). EV infection was confirmed in the one patient with cardiorespiratory failure. No EVs were isolated in the CSF of patients diagnosed with ANS dysregulation or encephalomyelitis; EV infection was later confirmed in these patients through cell cultures in nasopharyngeal fluids or feces (Table 4).

Pleocytosis was detected in the CSF, with a mean value of 192.82 leukocytes $/ \mathrm{mm}^{3}$ (IQR: 14-197), mainly neutrophils (44\%; IQR: 18-71\%) and lymphocytes (40\%; IQR: 15-60\%). CSF glucose and protein levels were normal in all cases.

In blood samples, 54\% patients ( $\mathrm{n}=94$ ) showed leukocytosis (mean value: 12449 x10E9/L [IQR: 941014770]) and 7\% had neutrophilia. High transaminase levels were detected in 34 cases (19.5\%).

Patients received symptomatic treatment and, when necessary, supportive treatment, consisting of intravenous immunoglobulin therapy $(n=9 ; 5.2 \%)$, corticosteroids $(n=6 ; 3.4 \%)$, and/or plasma exchange $(\mathrm{n}=1 ; 0.6 \%)$. 
All but one of the patients survived. Three patients — diagnosed with brainstem encephalitis, encephalitis, and ANS dysregulation, respectively-showed mild action tremor on follow-up. In all three cases, these conditions resolved within a few months. None of the other patients showed neurological symptoms after resolution of the infection. The one death in this series involved a preterm newborn with multisystemic involvement due to echovirus 11 (E-11) infection.

\section{Chronological and temporal distribution of genotypes}

During the study period, a mean of 17 cases were diagnosed annually. The largest number of cases $(n=37)$ was observed in 2012 (Figure 1). In terms of temporal distribution in incidence rates over the year, there were two peaks in the May-July period and November-December period (Figure 2).

EV genotyping was performed in 69 patients. Eleven different genotypes were identified, as follows: E-30 and EV-A71 (17 patients each, 50\%), coxsackievirus B5 $(\mathrm{n}=9)$ and echovirus 18 and 5 (E-18 and E-5; 6 patients each) (Figure 3).

EV-A71 was first observed in 2016 (12 cases), disappeared in 2017, and reappeared in 2018 and 2019, but with a much lower incidence. E-11 was detected from 2009 to 2011, but then disappeared. In the last two years of the study period (2018 and 2019), five new genotypes appeared: echovirus 6, 7, 9 and 13 (E-6, E7, E-9 and E-13) and coxsackievirus A6 (CV-A6). Only two genotypes-E-30 and CV-5-were detected in every year (Figure 4).

\section{Clinical and analytical characteristics according to EV genotype}

While all EV genotypes were associated with aseptic meningitis, the most common were E-30, CV-B5 and E-18 (Table 5).

In patients with encephalitic involvement (encephalitis, brainstem encephalitis, encephalomyelitis, or ANS dysregulation), the most common genotype was EV-A71 (Table 5). Seventeen patients (all aged from 6 months to 2 years) presented with EV-A71 infection; all of these patients (100\%) developed fever at onset. Two children had a seizure. Of these 17 patients with EV-A71, 13 had skin lesions, three had herpangina and four HFMD. Ten children required admission to the PICU: of these, two required mechanical ventilation and one received inotropic treatment.

EV-A71 was detected in the CSF in three of the 17 patients with this genotype; in the other 14 patients, the virus was detected in nasopharyngeal fluid or feces. Mean leukocyte counts were higher in patients with the more severe EV-A71 infection (Table 6).

In this series, the incidence of the E-30 and EV-A71 genotypes were equally common (Table 6). However, in contrast to EV-A71, E-30 was detected in all 11 years of the study. It is more common in those older than 2 years of age, and fever is almost constant (15/17). No skin lesions or respiratory symptoms were observed in children with E-30 infection, which was detected in the CSF of $100 \%$ of patients. Pleocytosis numbers (374 cells/camp) for this genotype were significantly than other genotypes. E-30 was detected in 16 patients with aseptic meningitis and one patient with brainstem encephalitis. 
The most commonly detected genotype in children $<6$ months of age was CV-B5, which was associated with respiratory symptoms in half of those patients but not with skin involvement. The mean duration of admission was longer in patients with CV-B5 infection (19 days; IQR: 0.4-37.6 days) respect other EV, probably due to the younger age of these patients.

Given that severe disease is usually associated with neurological involvement, we compared patients with meningism with and without neurological involvement (decreased consciousness, seizures, or signs of neurological involvement) in an effort to identify the risk factors associated with encephalitic involvement. The following variables were significantly associated with encephalitic involvement (Table 6): age between 2 and 5 years $(\mathrm{p}<0.001)$; time from onset to first neurological signs $(\mathrm{p}<0.001)$; presence of skin lesions ( $\mathrm{p}=0.012)$; HFMD ( $\mathrm{p}=0.025)$; and EV-A71 genotype $(\mathrm{p}<0.001)$.

\section{DISCUSSION}

The main aim of this study was to determine the risk factors for severe neurological involvement in children with EV infections. Our main findings were that the children with the highest risk of developing severe neurological involvement after EV infection were those 2 to 5 years of age who develop symptoms $<12$ hours from illness onset, especially if unspecific skin rash or HFMD are present.

$\mathrm{EV}$ infection is common in children. However, given that some EV infections can have serious consequences, early diagnosis is essential, which is why it is important to determine the clinical signs and genotypes most commonly associated with severe disease. This would allow clinicians to offer patients better treatment while also avoid unnecessary tests and preventing hospitalization.

In Europe, only a few studies have been performed before the year 2015 to evaluate the prevalence of different genotypes in patients with EV infections. A report from Italy based on wastewater samples showed that CV-B5 and E-6 were the predominant genotypes in the 2006-2010 period, and coxsackievirus B4 (CVB4) after 2010 [20]. In 2008, a multicenter study in Spain evaluated the EV genotypes in CNS infections, finding that the most prevalent EV genotypes were E-4, E-30, E-9, and E-6 [21]. In Asia, due to HFMD outbreaks with neurological involvement, more studies are found from 2010. Between 2010 and 2016, the EV-A71 and coxsackievirus A16 (CV-A16) were the two most common genotypes associated with HFMD in Shanghai, China [22]. Although that study reported a clear association between EV-A71 and worse clinical outcomes, the authors did not specify the type of neurological involvement, which makes it difficult to compare those findings to our results. Another Chinese study evaluated patients with HFMD in Sichuan between 2011 and 2017 [23], finding that the most prevalent genotype changed over time, from the EVA71 genotype in the early years to the CV-A16 genotype in the latter years. Until 2013, these are the most common genotypes isolated, but from this year to nowadays, there is an increase of other EV genotypes infection. However, those authors did not describe the type or extent of neurological involvement in the patients.

The number of published studies has increased in recent years, especially after the 2016 EV-A71 outbreak in Europe. One European study evaluated biological samples obtained in 2015-2017, finding that the most common genotypes changed over time, and showing that the most common EV in 2015 was E-6, while 
EV-D68 and EV-A71 were more prevalent in 2016, and E-30 and E-18 in 2017 [9]. It is important to note, however, that the data from that large series included all patients diagnosed with an EV infection, regardless of the clinical presentation. In our series, by contrast, we included only EV infections with neurological involvement, which is why some genotypes may be underrepresented in our series. The most common genotypes in our series by year were E-30 (2015), EV-A71 (2016), and E-18 and CV-B5 (2017).

In our series, we divided the patients into five age groups in order to evaluate genotypes and clinical presentation by age. In infants less than six months of age, the most common genotype was CV-B5, which was mainly associated with aseptic meningitis. In patients aged 6 months to 2 years, the most common genotype was EV-A71, which was associated with all types of neurological disease, but not with cardiorespiratory failure. Finally, in children up to 2 years of age, the most common neurological disease was aseptic meningitis and the most common genotype in this age group was E-30. Unlike EV-A71, neither CV-B5 nor E-30 were associated with skin rash or HFMD prior to onset of the neurological symptoms. CV-B5 was associated with respiratory symptoms in $50 \%$ of patients.

Previous studies have shown that EV-A71, unlike other genotypes, is rarely isolated in CSF (in 0-5\% of cases) [15-17,24]. In our series, this genotype was only detected in a few patients with aseptic meningitis, which is why if a EV infection is suspected in these patients, other biological samples, such as nasopharyngeal fluid or feces, should be sampled to check for the presence of enterovirus. We obtained a positive RT-PCR result in $69 \%$ of respiratory samples and in $62.9 \%$ of fecal samples. Some studies have found that detection rates are higher in fecal samples (up to $95 \%$ in an Australian study) than in nasopharyngeal samples (16\% in a European study and $85 \%$ in the aforementioned Australian study) $[9,25]$.

Clearly, if we could identify the patients most likely to develop severe neurological impairment this would help to optimize treatment. To this end, we classified the patients with HFMD and neurological involvement according to the WHO criteria established in 2011 (Table 6), which permits implementation of a management strategy based on symptom severity. For example, in our series, patients with aseptic meningitis were discharged after a few hours of monitoring in the emergency room; by contrast, patients showing decreased consciousness, and especially those with symptoms suggestive of brainstem encephalitis, were admitted to the ICU for closer monitoring to watch for ANS dysregulation or cardiorespiratory failure [26-30].

Once patients were classified according to these different clinical presentations, we evaluated the clinical, radiological, and analytical data to identify the risk factors associated with neurological involvement. Several variables - age between 2 and 5 years, time from infection onset, presence of skin rash, HFMD, and the EV-A71 genotype-were significantly associated with neurological involvement. Other studies have identified other risk factors, including the presence of leukocytosis at admission, age, male sex, and certain genetic risk factors [15] . A 2014 metanalysis found that a fever $>37.5^{\circ} \mathrm{C}$ lasting more than three days, age, lethargy, hyperglycemia, vomiting, neutrophilia, and the EV-A71 genotype were associated with severe neurological impairment related to HFMD [31]. 
Our findings indicate that the patients who appear to have a high risk of developing severe neurological involvement are those 2 to 5 years of age who develop symptoms $<12$ hours from illness onset, especially if skin rash or HFMD are present.

Another important finding of this study is the high prevalence of aseptic meningitis among infants $<3$ months of age. This is probably due to the fact that lumbar puncture is almost compulsory in infants under 1 month of age presenting with fever without a focus of infection, since it is nearly impossible to detect meningism at this age, and therefore some mildly symptomatic patients can be better diagnosed in this age group than in eldest children [32-34].

\section{Study strengths and limitations}

Despite the limitations of this retrospective study, we believe that the findings warrant additional research to more clearly define the risk factors associated with enterovirus infections in pediatric populations. Ideally, future studies should include a larger sample size with a multicentric design to confirm the findings of the present study.

\section{CONCLUSIONS}

In this retrospective series, several risk factors were associated with neurological involvement in pediatric patients with EV infection. In children between 2 to 5 years of age who develop neurological signsassociated with a non-specific skin rash or HFMD — within the first 12 hours from onset, a broad, early microbiological study should be considered. A lumbar puncture should be performed to check for the presence of an EV infection using RT-PCR. Other biological samples (feces or respiratory fluids) should also be collected for analysis. In cases with symptoms suggestive of brainstem encephalitis, cardiorespiratory monitoring is recommended to check for signs of severe neurological involvement such as lower cranial nerve palsy, arrhythmia, or respiratory distress. CSF should be evaluated in infants under 3 months of age who present fever without focus to check for aseptic meningitis, even if clinical signs of meningism are not present.

\section{REFERENCES}

1. Flores-Gonzalez JC, Jordan-Garcia I, Turon-Vinas E, Montero-Valladares C, Tellez-Gonzalez C, Fernandez-Carrion F, et al (2015). [Etiology, clinical presentation and outcome of severe viral acute childhood encephalitis (ECOVE study)]. Rev Neurol 61(1):7-13. PMID: 26108903

2. Ludlow M, Kortekaas J, Herden C, Hoffmann B, Tappe D, Trebst C, et al (2016). Neurotropic virus infections as the cause of immediate and delayed neuropathology. Acta Neuropathol 131(2):159-84. doi: 10.1007/s00401-015-1511-3.

3. Solomon T, Lewthwaite P, Perera D, Cardosa MJ, McMinn P, Ooi MH (2010). Virology, epidemiology, pathogenesis, and control of enterovirus 71. Lancet Infect Dis 10(11):778-90. doi: 10.1016/S1473-3099(10)70194-8. 
4. McWilliam Leitch EC, Cabrerizo M, Cardosa J, Harvala H, Ivanova OE, Kroes ACM, et al (2010). Evolutionary dynamics and temporal/geographical correlates of recombination in the human enterovirus echovirus types 9, 11, and 30. J Virol 84(18): 9292-300. doi: 10.1128/JVI.00783-10.

5. Karrasch M, Fischer E, Scholten M, Sauerbrei A, Henke A, Renz DM, et al (2016). A severe pediatric infection with a novel enterovirus A71 strain, Thuringia, Germany. J Clin Virol 84:90-5. doi: 10.1016/j.jcv.2016.09.007.

6. Tamura K, Stecher G, Peterson D, Filipski A, Kumar S (2013). MEGA6: Molecular Evolutionary Genetics Analysis version 6.0. Mol Biol Evol 30(12):2725-9. doi: 10.1093/molbev/mst197.

7. Martin NG, Iro MA, Sadarangani M, Goldacre R, Pollard AJ, Goldacre MJ (2016). Hospital admissions for viral meningitis in children in England over five decades: a population-based observational study. Lancet Infect Dis 16(11):1279-87. doi: 10.1016/S1473-3099(16)30201-8.

8. Tian X, Han Z, He Y, Sun Q, Wang W, Xu W, et al (2021). Temporal phylogeny and molecular characterization of echovirus 30 associated with aseptic meningitis outbreaks in China. Virol J 18(1):118. doi: 10.1186/s12985-021-01590-4.

9. Bubba L, Broberg EK, Jasir A, Simmonds P, Harvala H, et al (2020). Enterovirus study collaborators. Circulation of non-polio enteroviruses in 24 EU and EEA countries between 2015 and 2017: a retrospective surveillance study. Lancet Infect Dis 20(3): 350-61. doi: 10.1016/S1473-3099(19)30566-3.

10. Cui P, Li Y, Zhou CC, Zhou YH, Song CL, Qiu Q, et al (2019) [Clinical analysis of 555 outpatients with hand, foot and mouth diseases caused by different enteroviruses]. Zhonghua Er Ke Za Zhi 57(6):44551. doi: 10.3760/cma.j.issn.0578-1310.2019.06.009.

11. Hsia S-H, Lin J-J, Chan O-W, Lin T-Y (2020) Cardiopulmonary failure in children infected with Enterovirus A71. J Biomed Sci 27(1):53. doi: 10.1186/s12929-020-00650-1.

12. Gonzalez G, Carr MJ, Kobayashi M, Hanaoka N, Fujimoto T (2019). Enterovirus-Associated Hand-Foot and Mouth Disease and Neurological Complications in Japan and the Rest of the World. Int J Mol Sci 20(20): 5201. doi: 10.3390/ijms20205201.

13. World Health Organization, World Health Organization, Regional Office for the Western Pacific, Regional Emerging Diseases Intervention Centre. A guide to clinical management and public health response for hand, foot and mouth disease (HFMD). World Health Organization; 2011. https://iris.wpro.who.int/bitstream/handle/10665.1/5521/9789290615255_eng.pdf. Accessed 13 January 2021.

14. Pascual-Goñi E, Josa M, Launes C, Querol L, Del Cuerpo M, Bosch MA, Jordan I, Turón-Viñas E (2019). Excellent Response to Plasma Exchange in Three Patients With Enterovirus-71 Neurological Disease. Front Neurol 10:548. doi: 10.3389/fneur.2019.00548. 
15. Taravilla CN, Pérez-Sebastián I, Salido AG, Serrano CV, Extremera VC, Rodríguez AD, et al (2019). Enterovirus A71 Infection and Neurologic Disease, Madrid, Spain, 2016. Emerg Infect Dis 25(1). doi: 10.3201/eid2501.181089.

16. González-Sanz R, Casas-Alba D, Launes C, Muñoz-Almagro C, Ruiz-García MM, Alonso M, et al (2019). Molecular epidemiology of an enterovirus A71 outbreak associated with severe neurological disease, Spain, 2016. Euro Surveill 24(7): 1800089. doi: 10.2807/1560-7917.ES.2019.24.7.1800089.

17. Cabrerizo M, Tarragó D, Muñoz-Almagro C, Del Amo E, Domínguez-Gil M, Eiros JM, et al (2014). Molecular epidemiology of enterovirus 71, coxsackievirus A16 and A6 associated with hand, foot and mouth disease in Spain. Clin Microbiol Infect 20(3): O150-156. doi: 10.1111/1469-0691.12361.

18. Böttcher S, Diedrich S, Keeren K, The Laboratory Network For Enterovirus Diagnostic LaNED (2019). Increased detection of enterovirus A71 infections, Germany, 2019. Euro Surveill 24(39): 1900556. doi: 10.2807/1560-7917.ES.2019.24.39.1900556.

19. Messacar K, Spence-Davizon E, Osborne C, Press C, Schreiner TL, Martin J, et al (2020). Clinical characteristics of enterovirus A71 neurological disease during an outbreak in children in Colorado, USA, in 2018: an observational cohort study. Lancet Infect Dis 20(2): 230-9. doi: 10.1016/S14733099(19)30632-2.

20. Pellegrinelli L, Binda S, Chiaramonte I, Primache V, Fiore L, Battistone A, et al (2013). Detection and distribution of culturable Human Enteroviruses through environmental surveillance in Milan, Italy. J Appl Microbiol 115(5): 1231-9. doi: 10.1111/jam.12321.

21. Cabrerizo M, Trallero G, Echevarría JE, Moreno-Docón A, Pena MJ, Pérez-Ruiz M, et al (2010). Molecular characterization of enteroviruses associated with neurological infections in Spain, 2008. J Med Virol 85(11):1975-7. doi: 10.1002/jmv.23693.

22. Xu Y, Zheng Y, Shi W, Guan L, Yu P, Xu J, et al (2020). Pathogenic characteristics of hand, foot and mouth disease in Shaanxi Province, China, 2010-2016. Sci Rep 10(1): 989. doi: 10.1038/s41598-02057807-z.

23. Peng D, Ma Y, Liu Y, Lv Q, Yin F (2020). Epidemiological and aetiological characteristics of hand, foot, and mouth disease in Sichuan Province, China, 2011-2017. Sci Rep 10(1): 6117. doi: 10.1038/s41598-020-63274-3.

24. Ooi MH, Wong SC, Lewthwaite P, Cardosa MJ, Solomon T (2010). Clinical features, diagnosis, and management of enterovirus 71. Lancet NeuroL 9(11): 1097-105. doi: 10.1016/S1474-4422(10)70209X.

25. Teoh H-L, Mohammad SS, Britton PN, Kandula T, Lorentzos MS, Booy R, et al (2016). Clinical Characteristics and Functional Motor Outcomes of Enterovirus 71 Neurological Disease in Children. JAMA Neurol 73(3): 300-7. doi: 10.1001/jamaneurol.2015.4388. 
26. Lu H-K, Lin T-Y, Hsia S-H, Chiu C-H, Huang Y-C, Tsao K-C, et al (2004). Prognostic implications of myoclonic jerk in children with enterovirus infection. J Microbiol Immunol Infect 37(2): 82-7. PMID: 15181488.

27. Chang LY, Lin TY, Hsu KH, Huang YC, Lin KL, Hsueh C, et al (1999). Clinical features and risk factors of pulmonary oedema after enterovirus-71-related hand, foot, and mouth disease. Lancet 354(9191): 1682-6. doi: 10.1016/S0140-6736(99)04434-7.

28. Ooi MH, Wong SC, Mohan A, Podin Y, Perera D, Clear D, et al (2009). Identification and validation of clinical predictors for the risk of neurological involvement in children with hand, foot, and mouth disease in Sarawak. BMC Infect Dis 9: 3. doi: 10.1186/1471-2334-9-3.

29. Fu Y-C, Chi C-S, Lin N-N, Cheng C-C, Jan S-L, Hwang B, et al (2006). Comparison of heart failure in children with enterovirus 71 rhombencephalitis and cats with norepinephrine cardiotoxicity. Pediatr Cardiol 27(5): 577-84. doi: 10.1007/s00246-005-0915-6.

30. Fu Y, Chi C, Chiu Y, Hsu S, Hwang B, Jan S, et al (2004). Cardiac complications of enterovirus rhombencephalitis. Arch Dis Child 89(4): 368-73. doi: 10.1136/adc.2003.029645.

31. Fang Y, Wang S, Zhang L, Guo Z, Huang Z, Tu C, et al (2014). Risk factors of severe hand, foot and mouth disease: a meta-analysis. Scand J Infect Dis 46(7): 515-22. doi: $10.3109 / 00365548.2014 .907929$.

32. Mukherjee G, Waris R, Rechler W, Kudelka M, McCracken C, Kirpalani A, et al (2021). Determining Normative Values for Cerebrospinal Fluid Profiles in Infants. Hosp Pediatr 11(9): 930-6. doi: 10.1542/hpeds.2020-005512.

33. Nassrallah B, Bamberger E, Cohen S, Srugo I, Golan-Shany O, Shlonsky Y, et al (2021). The yield of CSF molecular testing in febrile neonates. Eur J Clin Microbiol Infect Dis 40(7): 1553-57. doi: 10.1007/s10096-021-04168-8.

34. Chen W, Dai S, Xu L (2021). Clinical characterization of benign enterovirus infection in neonates. Medicine (Baltimore) 100(18): e25706. doi: 10.1097/MD.0000000000025706.

\section{STATEMENTS AND DECLARATIONS}

\section{DECLARATIONS}

- Funding: The authors declare that no funds grants or other support were received during the preparation of thismanuscript.

- Competing interests: The authors have no relevant financial or non-financial interests to disclose.

- Availability of data and material: not applicable.

- Code availability: not applicable.

- Authors' contributions: All authors contributed to the study conception and desing. Material preparation 
was performed by ElisendaMoliner, Margarita del Cuerpo and Nuria Rabella. Data collection was performed by Fátima Castillo, Eulàlia Turón-Viñas, Elisenda Moliner, Laura Armendáriz and Emma Carbonell. Analysis was performedby Eulàlia Turón-Viñas and Elisenda Moliner. The first draft of the manuscript was written by Fátima Castillo and Eulàlia Turón-Viñas and all authors commented on previous versions of the manuscript. All authors read and approved the final manuscript.

- Ethics approval: This study was performed in line with the principles of the Declaration of Helsinki. Approval was grantedby the Ethics Committee of Hospital Sant Pau - Research Institute, Barcelona.

- Consent to participate: informed consent was obtained from tutors and parents.

- Consent for publication: informed consent was obtained from tutors and parents.

\section{TABLES AND FIGURES}

Table legends:

Table 1. Patient characteristics.

Table 2. Clinical presentation.

Table 3. Classification of patients according to WHO case definition.

Table 4. Enterovirus detection in cerebrospinal fluid (CSF) according to clinical presentation.

Table 5. Clinical presentation according to genotype.

Table 6. Risk factors for neurological involvement.

Figure legends:

Fig.1. Annual distribution of patients during the 11-year study period $(n=174)$.

Fig.2. Distribution of cases throughout the year $(n=174)$.

Fig.3. Isolated EV genotypes $(n=69)$.

Fig.4. Distribution of genotypes during the study period. 
Figures

Figure 1. Annual distribution of patients during the 11-year study period ( $\mathrm{n}=174)$.

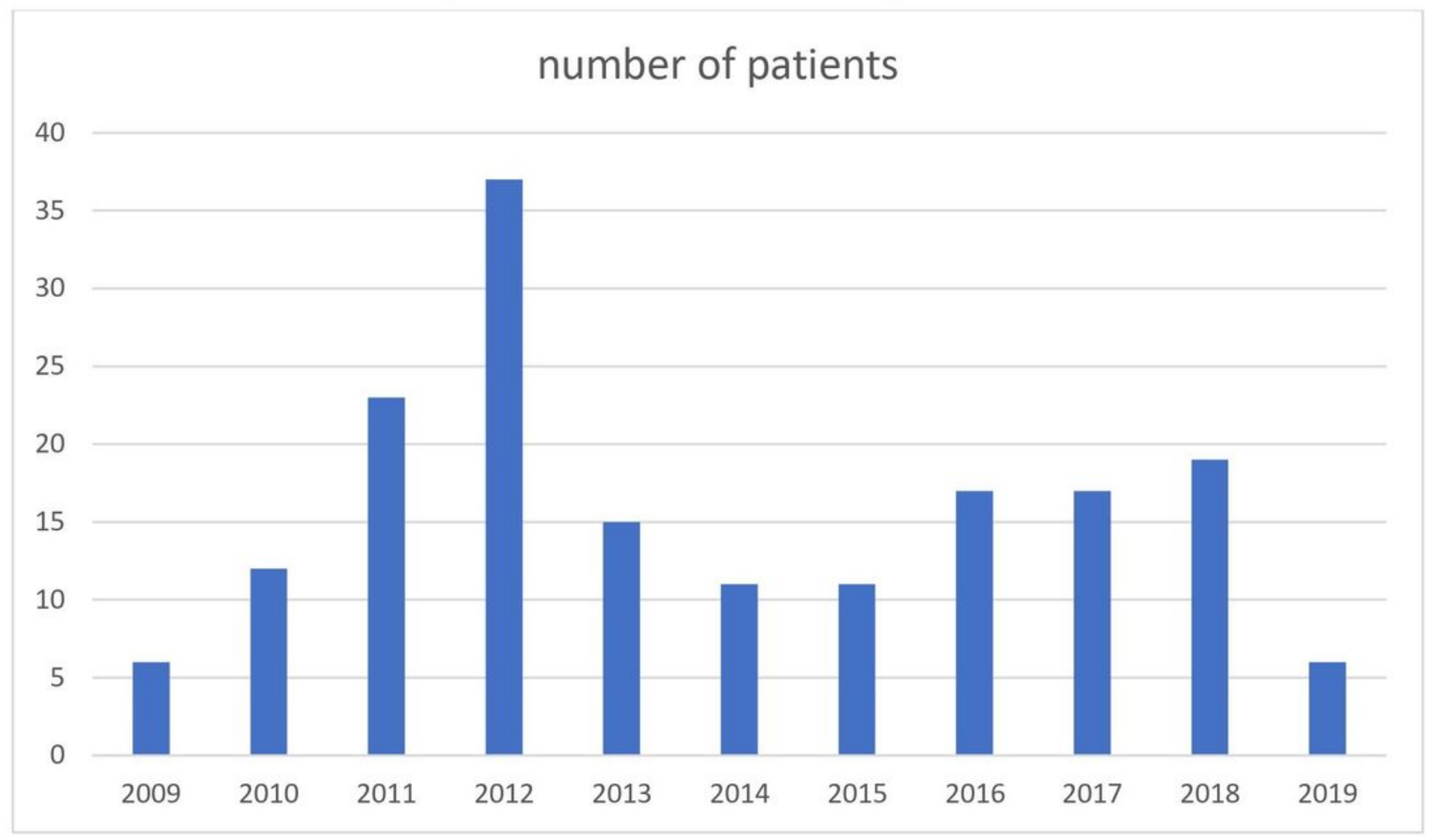

Figure 1

Please See image above for figure legend. 
Figure 2. Distribution of cases throughout the year $(n=174)$

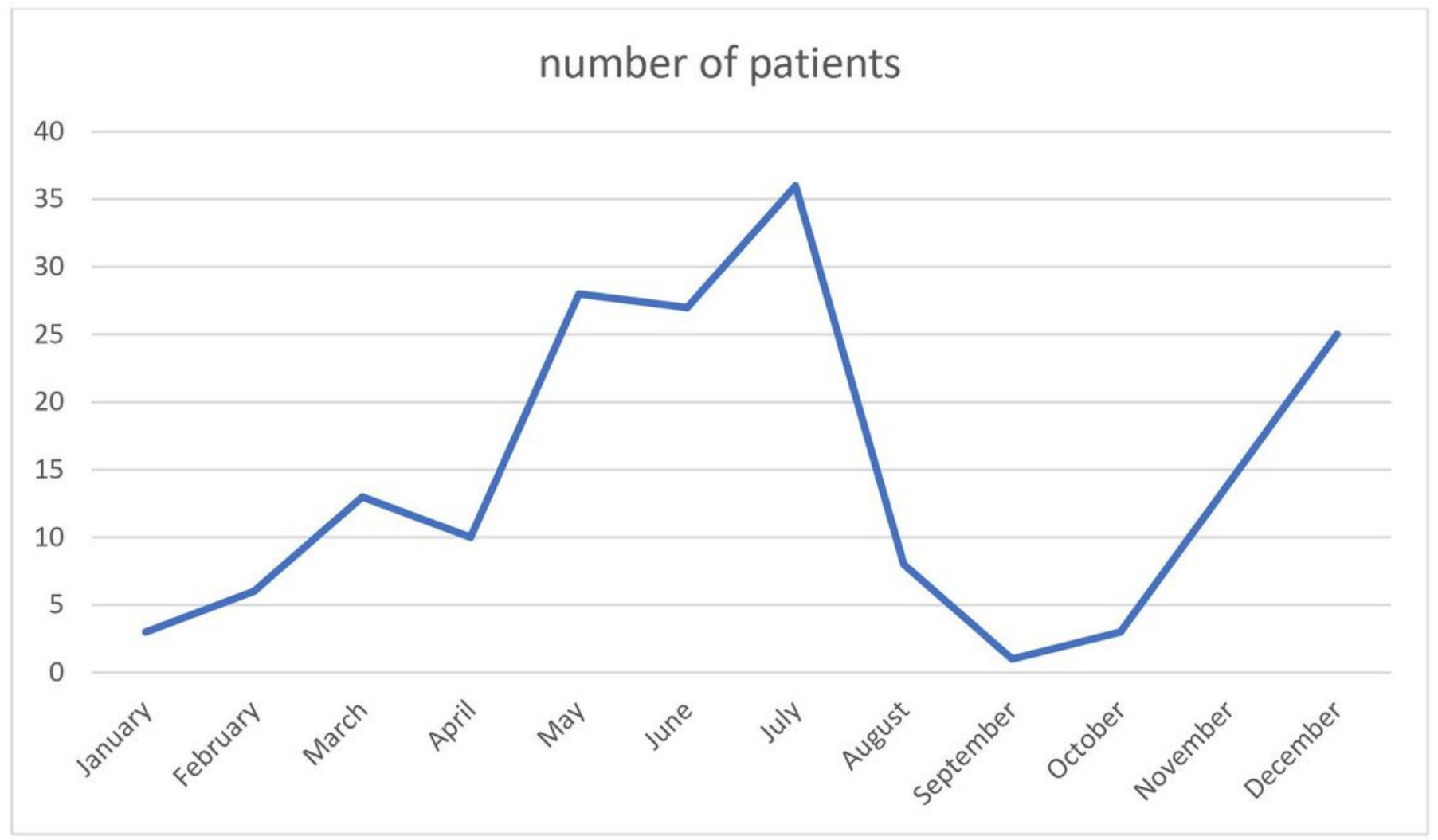

Figure 2

Please See image above for figure legend. 
Figure 3. Isolated EV genotypes $(\mathrm{n}=69)$.

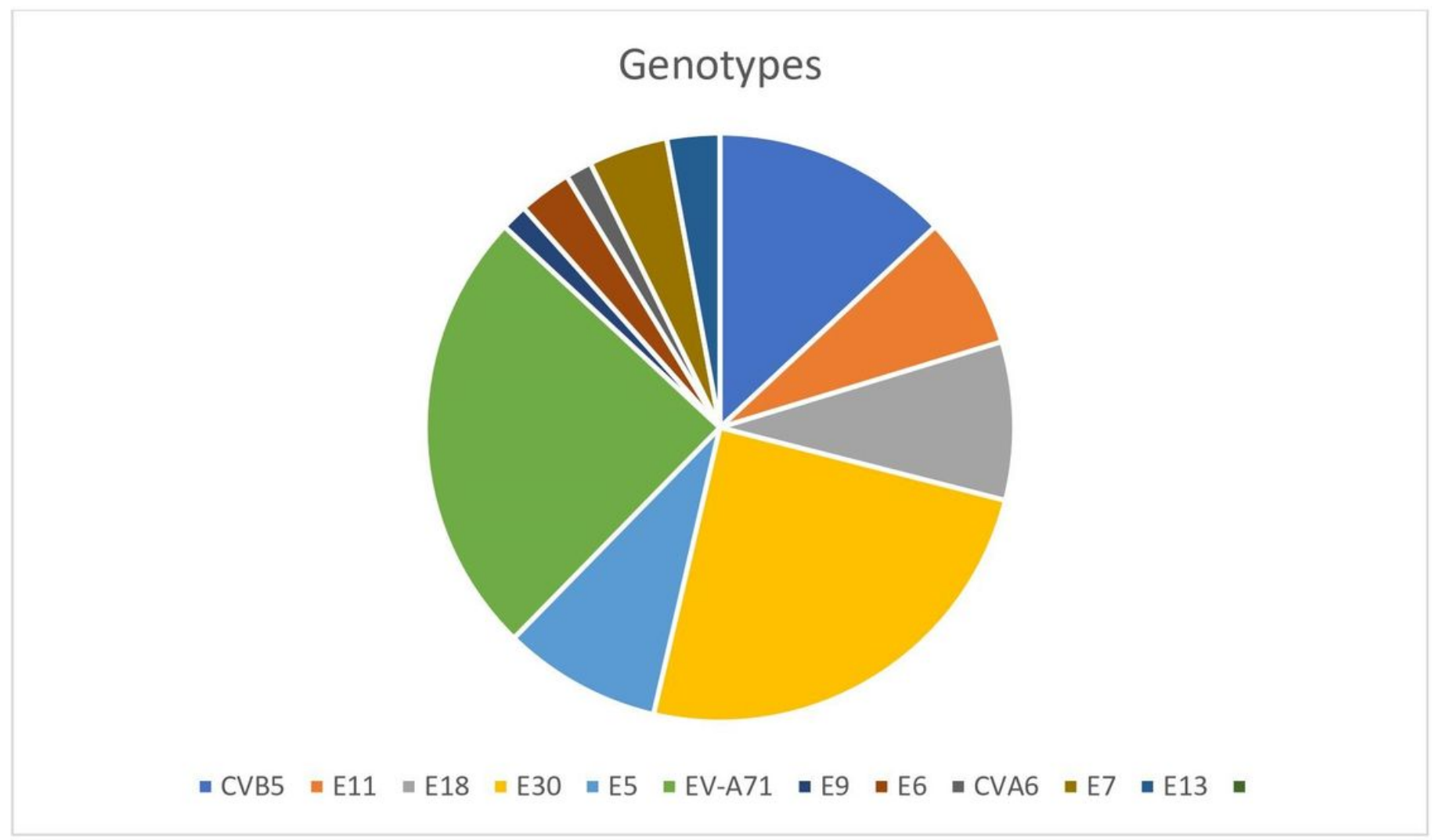

Figure 3

Please See image above for figure legend. 
Figure 4. Distribution of genotypes during the study period

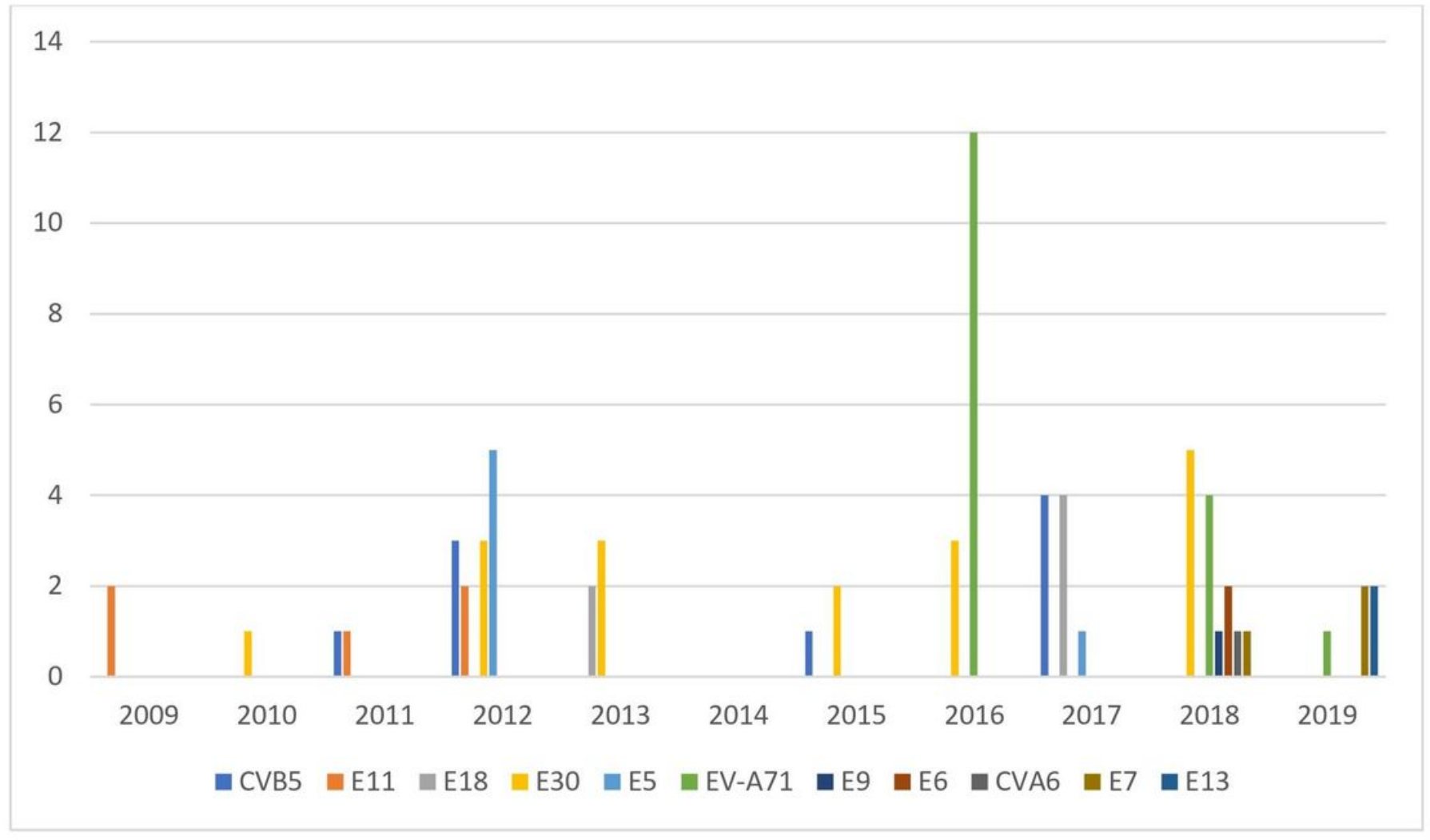

\section{Figure 4}

Please See image above for figure legend.

\section{Supplementary Files}

This is a list of supplementary files associated with this preprint. Click to download.

- Table1.Patientcharacteristics.pdf

- Table2.Clinicalpresentation.pdf

- Table3.Classificationofpatients.pdf

- Table4.EnterovirusdetectioninCSF.pdf

- Table5.Clinicalpresentationaccordingtogenotype.pdf

- Table6.Riskfactorsforneurologicalinvolvement.pdf 